\title{
Factorization theorems for exclusive heavy-quarkonium production
}

\author{
Geoffrey T. Bodwin, ${ }^{1}$ Xavier Garcia i Tormo, ${ }^{1}$ and Jungil Lee ${ }^{2}$ \\ ${ }^{1}$ High Energy Physics Division, Argonne National Laboratory, \\ 9700 South Cass Avenue, Argonne, Illinois 60439, USA \\ ${ }^{2}$ Department of Physics, Korea University, Seoul 136-701, Korea
}

(Dated: November 20, 2018)

\begin{abstract}
We outline the proofs of the factorization theorems for exclusive two-body charmonium production in $B$-meson decay and $e^{+} e^{-}$annihilation to all orders in perturbation theory in quantum chromodynamics. We find that factorized expressions hold up to corrections of order $m_{c} / m_{b}$ in $B$-meson decay and corrections of order $m_{c}^{2} / s$ in $e^{+} e^{-}$annihilation, where $m_{c}$ is the charm-quark mass, $m_{b}$ is the bottom-quark mass, and $\sqrt{s}$ is the $e^{+} e^{-}$center-of-momentum energy.

PACS numbers: 12.38.-t, 12.38.Bx, 14.40.Gx
\end{abstract}

The exclusive decays of $B$ mesons into a light meson plus a charmonium are significant tools for understanding the weak interactions. For example, they could provide new constraints on the Cabibbo-Kobayashi-Maskawa (CKM) matrix and enhance our understanding of the origins of $\mathrm{CP}$ violation. However, the effects of the strong interactions are significant in such processes and present an obstacle to achieving reliable theoretical calculations of the process rates. The exclusive production of double-charmonium in $e^{+} e^{-}$annihilation provides an arena in which to explore the mechanisms of charmonium production and the interplay between the perturbative and nonperturbative regimes of quantum chromodynamics (QCD). However, measurements of the cross sections for double-charmonium production by the Belle [1] and BABAR 2] Collaborations have spurred a reexamination of the theoretical predictions for those cross sections [3].

In theoretical computations of the rates for the exclusive decays of $B$ mesons into a light meson plus a charmonium and of the rates for the exclusive production of double-charmonium in $e^{+} e^{-}$annihilation, a crucial step is the separation of the effects of the strong interactions into short-distance, perturbatively calculable contributions and long-distance, inherently nonperturbative contributions. Such separations are usually embodied in factorization theorems. In the case of the exclusive decays of $B$ mesons into a light meson plus a charmonium, several factorization theorems have been posited [4, ,5, 6$]$. In the case of the exclusive production of double-charmonium in $e^{+} e^{-}$annihilation, factorization conjectures have generally been formulated in terms of nonrelativistic QCD (NRQCD) [3]. In this letter, we outline the proofs of the factorization theorems for these processes in QCD to all orders in perturbation theory.

We wish to show that the amplitudes for these processes can be written in a factorized form. The meaning of this statement, for the case of exclusive decays of $B$ mesons into a light meson plus a charmonium, is that the decay amplitude is decomposed into the sum of products of a $B$-meson-to-light-meson form factor and an amplitude for a charm-quark-antiquark $(c \bar{c})$ pair to be produced at short-distances in a color-singlet state and evolve into a charmonium. The form factor contains a term that can be decomposed into a convolution of a hard-scattering amplitude with $B$-meson and lightmeson light-cone amplitudes and a term that cannot be decomposed further. (These two terms are analogous to the two terms in the factorization formula in Eq. (4) of Ref. [4] for the case of decays to two light mesons.) The $c \bar{c}$-to-charmonium amplitudes can be further decomposed into a sum of products of long-distance NRQCD matrix elements times short-distance coefficients. We argue that this factorized form holds up to corrections of relative order $m_{c} / \sqrt{s}$, where $s=m_{b}^{2}$. This result was suggested previously in Ref. [4]. For the case of the exclusive production of double-charmonium in $e^{+} e^{-}$annihilation, factorization means that the production amplitude decomposes into a sum of products of a shortdistance amplitude and two amplitudes for a $c \bar{c}$ pair to be produced at short-distances in a color-singlet state and evolve into a charmonium. Again, the $c \bar{c}$-to-charmonium amplitudes can be further decomposed into a sum of products of long-distance NRQCD matrix elements times short-distance coefficients. We argue that this factorized form holds up to corrections of relative order $m_{c}^{2} / s$, where $\sqrt{s}$ is the center-of-momentum (CM) energy of the $e^{+} e^{-}$ pair. Although our analyses are for the specific cases of $B$ decay and $e^{+} e^{-}$annihilation, the techniques that we describe should apply to other exclusive quarkonium production processes and may also shed light on factorization in inclusive quarkonium production. We note that, because we consider exclusive two-body decays, we avoid the issues raised in Ref. 7] concerning light particles comoving with the charmonium and the issues raised in Ref. [8] concerning an additional heavy quark co-moving with the charmonium.

We carry out our analyses in the $B$-meson rest frame and in the CM frame of the $e^{+} e^{-}$pair, choosing the three-momentum of the light meson or one of the charmonia to be in the negative 3 direction and choosing the three-momentum of the other charmonium to be in 
the positive 3 direction. For a momentum $k$, we define light-cone momentum components $k^{ \pm}=\left(k^{0} \pm k^{3}\right) / \sqrt{2}$, $\boldsymbol{k}_{\perp}$. We model the $B$ meson as an on-shell active bottom quark, which participates in the electroweak interaction, and an on-shell spectator light antiquark, which does not participate in the electroweak interaction. We take the quark and antiquark to be in a color-singlet state. We take the bottom quark to have momentum $p_{b}$, with $p_{b}^{0}=m_{b}$ and all other components of momentum zero. We take the spectator quark to have momentum $p_{l}$, with $p_{l \mu} \sim \Lambda_{\mathrm{QCD}}$, the QCD scale. We neglect $\Lambda_{\mathrm{QCD}}$ and the difference between $m_{b}$ and the $B$-meson mass in comparison with $m_{b}$. Similarly, we model the light meson as an on-shell active light quark and an on-shell spectator light antiquark, with the quark and antiquark in a color-singlet state. We take the active-quark momentum to be $p_{k_{1}}=y p_{k}+q_{k}$ and the spectator-quark momentum to be $p_{k_{2}}=(1-y) p_{k}-q_{k}$, where $p_{k}$ is the light-meson momentum. $p_{k}^{-} \sim m_{b}, q_{k}^{-}=0, p_{k \perp}=0$, $q_{k \perp} \sim \Lambda_{\mathrm{QCD}}$, and $p_{k}^{+} \sim q_{k}^{+} \sim \Lambda_{\mathrm{QCD}}^{2} / m_{b}$. Finally, we model a charmonium as an on-shell $c \bar{c}$ pair in a colorsinglet state, with the momentum of the $c(\bar{c})$ equal to $p_{i}\left(\bar{p}_{i}\right)$. We take $p_{i}=P_{i} / 2+q_{i}$ and $\bar{p}_{i}=P_{i} / 2-q_{i}$, where $P_{i}$ is the charmonium momentum and $P_{i} \cdot q_{i}=0$. In the charmonium rest frame, $q_{i}$ has only spatial components, whose magnitudes are of order $m_{c} v$, where $v$ is the typical charm-quark velocity in the charmonium rest frame $\left(v^{2} \approx 0.3\right)$. In the $e^{+} e^{-}$CM frame or $B$ meson rest frame, $P_{1}^{+} \sim P_{2}^{-} \sim Q, P_{1 \perp}=P_{2 \perp}=0$, and $P_{1}^{-} \sim P_{2}^{+} \sim m_{c}^{2} / Q$, where $Q=\sqrt{s}$ is the large momentum scale. One can think of the on-shell amplitude that we use in our model as the on-shell perturbative QCD amplitude that is matched to a soft-collineareffective-theory amplitude in the case of the light meson, a heavy-quark-effective-theory amplitude in the case of the $B$ meson, and an NRQCD amplitude in the case of the charmonia.

The next step is to identify the regions of loop momenta that give contributions that are leading in powers of $Q$ when we dress the lowest-order decay and production amplitudes in these models with additional gluons. These leading momentum regions correspond to particular Feynman-diagram topologies [9, 10], which we describe below. The identification of leading regions has been discussed in detail in Ref. [11]. For a loop momentum $k$, the leading regions are the hard region, in which $k_{\mu} \sim Q$; the collinear-to-plus (minus) region, in which $\left|k^{+}\right| \gg\left|k^{-}\right|\left(\left|k^{-}\right| \gg\left|k^{+}\right|\right), k^{+} k^{-} \sim \boldsymbol{k}_{\perp}^{2} \ll Q^{2}$, and the soft region, in which $k^{+} \sim k^{-} \sim\left|\boldsymbol{k}_{\perp}\right| \ll Q$. The collinear regions correspond to the directions of the momenta of the final-state light meson and charmonia. We note that, in the case of $B$-meson decays, there is also a "semi-hard region" in which propagators are off shell by an amount of order $m_{b} \Lambda_{\mathrm{QCD}}$. We treat this semi-hard region as part of the hard region. The "Glauber" region is also leading in power counting [12, 13]. In this region, $\left|k^{+}\right| \ll\left|k_{\perp}\right|$,

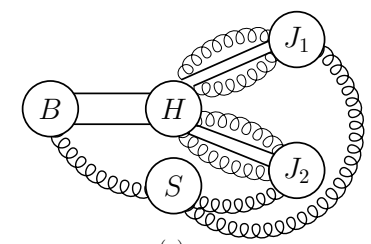

(a)

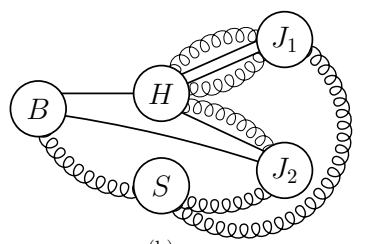

(b)
FIG. 1: Leading regions for the decay of a $B$ meson to a light meson and a charmonium.

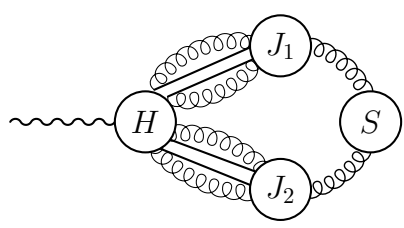

FIG. 2: Leading regions for double-charmonium production in $e^{+} e^{-}$annihilation. The wavy line represents the virtual photon.

$\left|k^{-}\right| \ll\left|k_{\perp}\right|, k_{\perp}^{2} \ll Q^{2}$. However, for the exclusive processes we are considering here, the contours of integration of loop momenta are not pinched in the Glauber region, and it is possible to deform them out of it on a diagramby-diagram basis. This is in contrast with the situation in, for instance, the Drell-Yan process, for which such a diagram-by-diagram contour deformation is not possible [11, 13, 14]. Therefore, we ignore the Glauber region in the remainder of our discussion. Contributions from the hard (semi-hard) region involve propagator denominators that are of order $s\left(m_{b} \Lambda_{\mathrm{QCD}}\right)$ and can be calculated in perturbation theory. Integrations of gluon momenta over the soft region or a collinear region that is associated with a massless particle encounter singularities in propagator denominators that result in logarithmic divergences. [The soft singularities are at $k_{\mu}=0$, and the collinear singularities are at $k^{-}=0, \boldsymbol{k}_{\perp}=0\left(k^{+}=0, \boldsymbol{k}_{\perp}=0\right)$ for the collinear-to-plus (to-minus) region.] Thus, contributions from the soft region and the collinear regions that are associated with a massless particle cannot be computed reliably in perturbation theory. The essence of the proofs of the factorization theorems that we consider here is to show that these contributions cancel or can be absorbed into the $B$-meson-to-light-meson form factor or the nonperturbative NRQCD matrix elements. When a gluon three-momentum becomes parallel to the threemomentum of a charm quark or charm antiquark, the potential collinear singularity is shielded by $m_{c}$. Hence, the contributions from such collinear regions can be computed reliably in perturbation theory. Nevertheless, it is important for the arguments that we will make below to treat these regions separately from $H$. As we shall see, the contributions from these regions cancel.

Now let us specify the diagrammatic topologies that 
correspond to the leading regions. We work in the Feynman gauge. There are two distinct topologies in the case of $B$-meson decays: one in which the $B$-meson and light-meson spectators participate in the hard interaction and another in which they do not. These topologies are shown in Figs. 1(a) and 1(b), respectively. After one has achieved factorization, the contribution from the first topology can be decomposed into an expression that contains the convolution of a hard-scattering amplitude with $B$-meson and light-meson light-cone amplitudes, while the contribution from the second topology cannot be decomposed further. The topology for the case of doublecharmonium production is represented in Fig. 2. The components of the topologies are as follows: a $B$ meson $(B)$; jet sub-diagrams for each of the collinear regions, corresponding to a charmonium $\left(J_{1}\right)$ and a light meson $\left(J_{2}\right)$ in Fig. 1 and the two charmonia $\left(J_{1}\right.$ and $\left.J_{2}\right)$ in Fig. 2, a hard sub-diagram $(H)$ that includes the lowest-order annihilation or production process; and a soft sub-diagram $(S)$. In $H$, all propagator denominators are of order $s$ or $m_{b} \Lambda_{\mathrm{QCD}} . \quad J_{i}$ contains the active- and spectator-quark lines for a given meson or charmonium, as well as gluons and loops involving quarks and ghosts with momenta collinear to the meson or charmonium. $J_{i}$ attaches to $H$ through the active- and spectator-quark lines in the topology of Fig. 1(a), through the activequark lines in the topology of Fig. 1(b) and through any number of gluons. $S$ includes gluons with soft momenta and loops involving quarks and ghost with soft momenta. $S$ attaches to $J_{i}$ and to the $B$-meson quark and antiquark lines through any number of soft gluon lines. Recall that the $B$-meson spectator antiquark itself carries a soft momentum. In Fig. 1(b), the light-antiquark-spectator line carries a momentum with minus component of order $\Lambda_{\mathrm{QCD}}$. Such a momentum can arise either through an endpoint contribution, in which $p_{k_{2}}^{-} \sim \Lambda_{\mathrm{QCD}}$ [4, 15], or through the soft-collinear-messenger mechanism [16], in which an emitted gluon carries away most of $p_{k_{2}}$.

At this point we can outline the proof of factorization. Suppose that a gluon from $J_{i}$ enters $H$. Then we can apply a collinear approximation to that gluon [11, 13, 14]. Specifically, we replace $g_{\mu \nu}$ in the gluonpropagator numerator with $k_{\mu} \bar{n}_{i \nu} / k \cdot \bar{n}_{i}$, where $k$ is the gluon momentum, $\bar{n}_{1}\left(\bar{n}_{2}\right)$ is a unit light-like vector in the minus (plus) direction. The index $\mu$ corresponds to the attachment of the gluon to $H$, and the index $\nu$ corresponds to the attachment of the gluon to $J_{i}$. For $k$ in the collinear region, the collinear approximation is valid at leading order in $Q$. Having made the collinear approximation, one can use the factor $k_{\mu}$ and the diagrammatic Ward identity (Feynman identity) for fermion lines $k \cdot \gamma=[(q+k) \cdot \gamma+m]-[q \cdot \gamma+m]$ and its generalizations to non-Abelian gauge theories to show that the gluons with collinear momenta decouple from $H$ 11, 13, 14]. In general, the decoupled gluons attach to light-like eikonal lines (path integrals of the gauge field) that connect to the point at which an active parton enters $H$. However, in our case these eikonal lines cancel because the light meson and the charmonia are color-singlet states. Now suppose that a gluon from $S$ attaches to a jet sub-diagram at a line with momentum $p$. We can apply a soft approximation to that gluon [17]. Specifically, we replace $g_{\mu \nu}$ in the gluon-propagator numerator with $k_{\mu} p_{\nu} / k \cdot p$, where $k$ is the gluon momentum, the index $\mu$ corresponds to the attachment of the gluon to $J_{i}$ and the index $\nu$ corresponds to the attachment of the gluon to $S$. We would like to use the factor $k_{\mu}$ in the soft approximation and the diagrammatic Ward identities to decouple the soft gluons. However, in general, the momentum $p$ is different for every line to which the soft gluon attaches. That is, the soft approximation, unlike the collinear approximation, is not independent of the line to which the $\mu$ end of the gluon attaches. Nevertheless, we can apply the same soft approximation to all lines in the collinear-to-lightmeson jet, since they are all proportional to the same light-like vector (up to corrections of order $\Lambda_{\mathrm{QCD}} / m_{b}$ ). For the constituents of each charmonium, we can also apply the same soft approximation up to corrections of order $m_{c} / Q$. The reason for this is that, in going from the rest frame of the charmonium to the $e^{+} e^{-} \mathrm{CM}$ frame or $B$-meson rest frame, the momenta of constituents of the charmonium undergo boosts that render all of the momenta nearly parallel. When we explicitly consider those boosts, it follows that, for the charmonium with momentum $P_{1}\left(P_{2}\right)$, the plus (minus) components are boosted by a factor of order $Q / m_{c}$ and the minus (plus) components are boosted by a factor of order $m_{c} / Q$, while the transverse components are unchanged. Thus, all of the momenta of the constituents of the charmonium with momentum $P_{1}\left(P_{2}\right)$ are dominated by the plus (minus) component, up to corrections of order $m_{c} / Q$. This result holds provided that all of the components are of approximately the same size in the charmonium rest frame. This is the case for $P_{i}, q_{i}$, but it is also true for other momenta that characterize the charmonium in its rest frame, such as a typical potential-gluon momentum (which has spatial components of order $m_{c} v$ and a temporal component of order $\left.m_{c} v^{2}\right)$ or a typical rest-frame soft-gluon momentum (which has all components of order $m_{c} v$ ). Therefore, we use a modified soft approximation for the constituents of the charmonium in the jet $J_{i}$ in which we replace $g_{\mu \nu}$ in the gluon-propagator numerator with $k_{\mu} n_{i \nu} / k \cdot n_{i}$, where $n_{1}\left(n_{2}\right)$ is a vector with unit component in the plus (minus) direction and all other components equal to zero. This modified soft approximation differs from the standard soft approximation for each constituent of a quarkonium by terms that are suppressed as $m_{c} / Q$. Hence, it accounts for all soft (logarithmic) singularities, up to terms that are suppressed as $m_{c} / Q$. We can use the modified soft approximation, plus the diagrammatic Ward identities to decouple the gluons with soft momenta from the charmonium jets, relying on the fact that the 
$c$ and $\bar{c}$ in each charmonium are in a color-singlet state [14, 17, 18]. Once the gluons with momenta collinear to the jet have been decoupled from $H$ and the gluons with soft momenta have been decoupled from the charmonium jets, there remain gluons with momenta collinear to the jet that begin and end within the jet. In the rest frame of the charmonium, these gluons correspond to soft and potential gluons. The contributions from regions of momentum of order $m_{c} v$ or less that arise from these gluons (including infrared divergences) can be absorbed into nonperturbative NRQCD matrix elements [19]. The associated NRQCD short-distance coefficients then contain only contributions involving momenta of order $m_{c}$. The NRQCD short-distance coefficients couple to the production process only through the active $c$ and $\bar{c}$ lines. Thus, we have arrived at the factorized form.

As we have mentioned, the modified soft approximation allows us to decouple gluons with soft momentum from the charmonium jets, up to corrections that are suppressed as $m_{c} / Q$. Thus, in general, one would expect corrections to the factorized form to appear at order $m_{c} / Q$. However, in the case of double-charmonium production in $e^{+} e^{-}$annihilation, $S$ decouples from both the collinear-to-plus charmonium jet and the collinearto-minus charmonium jet. Each decoupling holds up to corrections of order $m_{c} / Q$, and, so, the overall decoupling holds up to corrections of order $m_{c}^{2} / s$. In perturbation theory, the factorization-violating corrections may be enhanced by logarithms of $s / m_{c}^{2}$. Furthermore, they are infrared divergent. In reality, these infrared divergences are cut off by nonperturbative effects associated with confinement. Our analysis does not determine the size of these factorization-violating corrections: it shows only that they are proportional to one or two powers of $m_{c} / Q$. The constant of proportionality might be determined through experiment and/or lattice calculations.

At the lowest order in $\alpha_{s}$ and $v$, the decoupling of soft gluons from each charmonium is exact. At the lowest order in $\alpha_{s}$, the charmonium has only the $c$ and $\bar{c}$ as constituents. At the lowest order in $v$, one sets $q_{i}=0$, and the $c$ and $\bar{c}$ momenta become equal. Then, one can apply the same soft approximation for both the $c$ and $\bar{c}$ in the charmonium. Since, at the lowest order in $\alpha_{s}$, there is, at most, one soft gluon, the soft decoupling in double-charmonium production is exact if either charmonium is treated at the lowest order in $v$. An explicit calculation of the one-loop corrections to $S$-wave charmonium production in $B$-meson decays at the lowest order in $v$ [5] and an explicit calculation of the one-loop corrections to $\sigma\left[e^{+} e^{-} \rightarrow J / \psi+\chi_{c J}\right]$, in which the $J / \psi$ is treated at lowest order in $v$ [20], confirm our expectation that these corrections should be free of infrared divergences. $P$-wave charmonium production in $B$-meson decays was considered at the one-loop level in Ref. 21] within the factorization framework of Ref. [4]. When we use the light-cone distribution amplitudes that are spec- ified in Ref. [4], we obtain agreement with the calculations in Ref. [21], which yield only infrared divergences that are suppressed as $m_{c}^{2} / s$. As we have discussed, we find more generally that the violations of factorization are suppressed as $m_{c} / \sqrt{s}$. At higher orders in $v$, we expect the soft cancellation for each charmonium to hold only up to corrections of order $m_{c} / \sqrt{s}$, even in the case of $S$-wave charmonium production. Similarly, in relative order $\alpha_{s}^{2}$ and beyond, we expect the soft cancellation for each charmonium to hold only up to corrections of order $m_{c} / \sqrt{s}$, even at the lowest order in $v$.

We thank Kuang-Ta Chao, Jianwei Qiu, George Sterman, and Yu-Jie Zhang for helpful discussions. The work of G.T.B. and X.G.T. was supported by the U.S. Department of Energy, Division of High Energy Physics, under contract DE-AC02-06CH11357. J.L. was supported by KOSEF under contract R01-2008-000-10378-0.

[1] K. Abe et al. [Belle Collaboration], Phys. Rev. D 70, 071102 (2004).

[2] B. Aubert et al. [BABAR Collaboration], Phys. Rev. D 72, 031101 (2005).

[3] For a discussion of the current status of the theoretical predictions that are based on the NRQCD factorization approach, see G. T. Bodwin, J. Lee, and C. Yu, Phys. Rev. D 77, 094018 (2008).

[4] M. Beneke et al., Nucl. Phys. B 591, 313 (2000).

[5] J. Chay and C. Kim, arXiv:hep-ph/0009244.

[6] C. Bobeth, B. Grinstein, and M. Savrov, Phys. Rev. D 77, 074007 (2008).

[7] G. C. Nayak, J. W. Qiu, and G. Sterman, Phys. Lett. B 613, 45 (2005); Phys. Rev. D 72, 114012 (2005).

[8] G. C. Nayak, J. W. Qiu, and G. Sterman, Phys. Rev. Lett. 99, 212001 (2007); Phys. Rev. D 77, 034022 (2008).

[9] G. Sterman, Phys. Rev. D 17, 2773 (1978).

[10] G. Sterman, Phys. Rev. D 17, 2789 (1978).

[11] J. C. Collins, D. E. Soper, and G. Sterman, Adv. Ser. Direct. High Energy Phys. 5, 1 (1988).

[12] G. T. Bodwin, S. J. Brodsky, and G. P. Lepage, Phys. Rev. Lett. 47, 1799 (1981).

[13] G. T. Bodwin, Phys. Rev. D 31, 2616 (1985) [Erratumibid. D 34, 3932 (1986)].

[14] J. C. Collins, D. E. Soper, and G. Sterman, Nucl. Phys. B 261, 104 (1985).

[15] V. L. Chernyak and I. R. Zhitnitsky, Nucl. Phys. B 345, 137 (1990).

[16] T. Becher, R. J. Hill, and M. Neubert, Phys. Rev. D 69, 054017 (2004).

[17] J. C. Collins and D. E. Soper, Nucl. Phys. B 193, 381 (1981) [Erratum-ibid. B 213, 545 (1983)].

[18] J. C. Collins, D. E. Soper, and G. Sterman, Nucl. Phys. B 308, 833 (1988).

[19] G. T. Bodwin, E. Braaten, and G. P. Lepage, Phys. Rev. D 51, 1125 (1995) [Erratum-ibid. D 55, 5853 (1997)].

[20] Y. J. Zhang, Y. Q. Ma, and K. T. Chao, arXiv:0802.3655 [hep-ph].

[21] Z. Z. Song et al., Phys. Rev. D 69, 054009 (2004). 